\section{Peter Wehling}

\section{Postindustrialismus - eine ökologische Utopie?}

Mit den Theorien der postindustriellen Dienstleistungsgesellschaft werden seit ihrer Entstehung optimistische Einschätzungen der zukünftigen sozialen Entwicklung verbunden. Symptomatisch dafür ist das grundlegende Buch des französischen Ökonomen Jean Fourastié aus dem Jahre 1949: Die große Hoffnung des 20. Jahrhunderts. Gehofft wurde in den mittlerweile klassischen Entwürfen der postindustriellen Gesellschaft von Fourastié oder Daniel Bell - aber auch im kritischen Gegenkonzept von Alain Touraine - vor allem auf eine umfassende Humanisierung der Gesellschaft im Zuge der »Tertiarisierung «, des Übergangs zu den Dienstleistungen als dominierender gesellschaftlicher Tätigkeitsform. Dann nämlich bestünde das grundlegende Prinzip der sozialen Aktivität nicht mehr in der Bearbeitung der Natur, sondern in der Kommunikation zwischen Menschen. Zugleich sollte die wachsende Bedeutung des »theoretischen Wissens « die industriell-kapitalistische Klassenstruktur aufweichen und sie mittels einer allgemeinen Bildungsexpansion in eine gerechte, nur auf individueller Leistung beruhende soziale Ordnung transformieren (vgl. Bell 1975).

In den 80er Jahren wurden neue Erwartungen an die nachindustrielle Gesellschaft herangetragen: Aus der Tatsache, daß der Dienstleistungsbereich in allen industrialisierten Ländern nach 1945 schnell und stark expandiert war, wurde zum einen die Erwartung abgeleitet, der tertiäre Sektor könne in Zukunft die durch steigende Produktivität bei gleichzeitig relativ stagnierender industrieller Produktion freigesetzten Arbeitskräfte aufnehmen. Zumal Fourastié $(1954,137)$ hatte von einem Maximum von 80 Prozent tertiär Beschäftigter gegenüber jeweils zehn Prozent im primären, landwirtschaftlichen und im sekundären, industriellen Sektor gesprochen - eine Relation, die noch in keiner Gesellschaft bisher erreicht ist. Je schärfer und dauerhafter sich zum anderen die modernen Gesellschaften mit ökologischen Selbstgefährdungen konfrontiert sahen, desto mehr wurde der Gedanke bemüht, der Übergang zur nachindustriellen Gesellschaft werde die »Umweltkrise« der Industriegesellschaften wenn nicht überwinden, so doch deutlich abschwächen. Denn im Gegensatz zur industriellen Waren-

PROKLA. Zeitschrift für kritische Sozialwissenschaft, Heft 93, 23. Jg. 1993, Nr. 4, 664-683 produktion verbrauche die Erbringung von Diensten weder natürliche Rohstoffe noch verschmutze sie die Umwelt durch Emissionen oder Abfälle.

Ausgehend von einer kritischen Rekonstruktion der postindustriellen Argumentation werden im folgenden die theoretische Konsistenz und die empirische Plausibilität solcher Visionen (und damit zusammenhängend auch die Aussagekraft der volkswirtschaftlichen Sektorentheorie) überprüft. Es soll gezeigt werden, daß die Theorie des sektoralen Wandels zur nachindustriellen Gesellschaft ein zwar einfluBreiches, aber auf spezifische Weise selektives Deutungsmuster der industriellen Entwicklung nach 1945 darstellt - ein »historisches Modell« (Burkart Lutz), das so lange plausibel erschien, wie die zugrundegelegten gesellschaftlichen Trends ungebrochen wirksam waren und ihre problematischen Folgen noch nicht sichtbar oder offentlich thematisiert wurden:

»Wer .. auf der Grundlage des Fourastiéschen Drei-Sektoren-Modells argumentiert, ist der Mühe enthoben, danach zu fragen, was während des Industrialisierungs- und Modernisierungsprozesses in und mit den traditionellen Wirtschafts- und Lebensformen geschieht, deren fortschreitender Niedergang unverzichtbar erscheint, um für die historisch neuen Sektoren der Volkswirts

Expansion der modernen, industriell-kapitalistischen Sektoren gerichtet .. (Lutz, 1989, 281).

Ebensowenig stellt sich im Rahmen dieses »historischen Modells« die Frage, welche Auswirkungen auf die natürliche Umwelt der kontinuierliche Produktivitätsfortschritt im industriellen (und auch im landwirtschaftlichen) Sektor haben würde, der die Dienstleistungsgesellschaft erst möglich machen soll, (vgl. ebd., 233).

\section{Auf dem Weg in die Dienstleistungsgesellschaft?}

Die Theorien der postindustriellen Gesellschaft haben ihren Ursprung in den ersten Versuchen einer Einteilung der Volkswirtschaften in die »Sektoren« Landwirtschaft, Industrie und Dienstleistungen durch A.G.B. Fisher und C. Clark in den dreißiger und vierziger Jahren. Ihre wirtschaftstheoretische Begründung fand die Postindustrialismus-Theorie 1949 durch Fourastié. Sie beruhte auf einer mehr oder weniger linearen Fortschreibung der mutmaßlichen grundlegenden Trends der industriell-kapitalistischen Entwicklung, insbesondere des technischen Fortschritts in der industriellen Produktion als des entscheidenden dynamischen Elements des sozialen Wandels. Die Auswirkungen dieser Modernisierungsdynamik wurden in der Perspektive des Postindustrialismus verengt auf Verschiebungen der Nachfrage und der Beschäftigung zwischen Landwirtschaft, Industrie und Dienstleistungen. Aus dem teils empirisch festgestellten, teils theoretisch abgeleiteten und als säkular unterstellten Wandel zu den Dienstleistungen 
wurden dann tiefgreifende Änderungen der Sozialstruktur und der Formen der gesellschaftlichen Reproduktion abgeleitet.

Dienstleistungen und tertiärer Sektor wurden zunächst rein negativ definiert: So rechnete etwa Clark dem tertiären Sektor all die verbleibenden ökonomischen Aktivitäten zu, die nicht der Landwirtschaft oder der Industrie zugehören. Erst Fourastié entwickelte nach dem Grad des jeweiligen Produktivitätsfortschritts ein einheitliches Schema der Abgrenzung und Klassifikation der Sektoren - ohne daß damit aber die bloß negative und residuale Bestimmung des tertiären Sektors überwunden wäre. Denn da der primäre, landwirtschaftliche Sektor durch mäßigen technischen Fortschrití gekennzeichnet sei, und der sekundäre, industrielle Sektor die Bereiche mit großem technischem Fortschritt umfasse, verbleiben dem tertiären Sektor damit »sämtliche übrigen Wirtschaftstätigkeiten, d.h. die Wirtschaftsbereiche mit geringem oder ohne technischen Fortschritt《 (Fourastié 1954, 79f.). Diese Annahme eines schwachen Produktivitätsfortschritts im tertiären Sektor bildet bis heute eine der entscheidenden wirtschaftstheoretischen Grundlagen der Postindustrialismus-Konzepte.

In ihrer anspruchsvollsten Variante bei Bell, der »bisher letzten größeren bürgerlichen Gesellschaftstheorie der entwickelten Industriegesellschaften (Jänicke 1985, 239), basiert die Theorie der nachindustriellen Gesellschaft schließlich auf gleichgerichteten Trendannahmen in fünf Dimensionen (Bell 1975, 32):

»l. Wirtschaftlicher Sektor: der Übergang von einer güterproduzierenden zu ejner Dienstleistungswirtschaft:

2. Berufsstruktur: der Vorrang einer Klasse professionalisierter und technisch qualifizierter 2. Beruf

3. Axiales Prinzip: die Zentralität theoretischen Wissens als Quelle von Innovationen und Ausgangspunkt der gesellschaftlich-politischen Programmatik;

4. Zukunftsorientierung: die Steuerung des technischen Fortschritts und die Bewertung de Technologie;

5. Entscheidungsbildung: die Schaffung einer neuen 'intellektuellen Technologie'.«

Als charakteristisch für eine nachindustrielle Gesellschaft begriff Bell vor allem die Zunahme der Dienstleistungsberufe in den Bereichen Gesundheit, Erziehung und Bildung, Forschung und Verwaltung sowie die Ausbreitung einer neuen postindustriellen »Intelligentsia«.

Auf diese Annahmen stiitzt sich die These vom wesentlich humanen Charakter der postindustriellen Gesellschaft, die auf der Dienstleistung als einer Beziehung zwischen Menschen beruhe. Grundlegend für die nachindustrielle Gesellschaft ist es laut Bell (ebd., 168), »daß die Individuen (...) miteinander reden, statt auf eine Maschine zu reagieren«. Während unter der Dominanz der Industrie die Menschen »wie Sachen behandelt « würden, stehe im Zentrum der Dienstleistungsgesellschaft die »Begegnung oder Kommunikation und die Reaktion des Ich auf den Anderen «. Da sie auf einem »Spiel zwischen Personen $\ll$ beruhe, werde die nachindustrielle Gesellschaft eine »kommunale Gesellschaft $\ll$ sein (ebd., 136).

Doch vor allen Detailproblemen bleibt es das grundlegende Dilemma aller Theorien der Dienstleistungsgesellschaft, daßs es ihnen an einer einheitlichen und positiven Definition von Dienstleistungen mangelt (Gross 1983, 13). Bei Clark wie bei Fourastié wurden Dienstleistungen nur residual bestimmt, als die Tätigkeitsbereiche, die weder $\mathbb{L}$ andwirtschaft noch Industrie sind bzw. dem technischen Fortschritt nicht oder kaum zugänglich sind. Auch andere Abgrenzungsvorschläge von Diensten als Nicht-Sachgüter, nicht-lagerfähig, nicht-materiell, nicht-transportähig etc. bieten weder ein klares und einheitliches noch ein positives Kriterium. I Schon dieses Fehlen einer kategorialen Bestimmung von Dienstleistung demontiert im Grunde die »Tragfähigkeit des Begriffs der Dienstleistungs-Gesellschaft als gesellschaftstheoretischer Kategorie«(Baethge/Oberbeck 1986, 19).

\section{2. $\mathbb{Z}$ wei Begrïndungswersuche der Tertiarisierung}

Die These vom kontinuierlichen Wachstum des Dienstleistungssektor stützt sich auf zwei bereits von Fourastié formulierte Begründungen - eine nachfragetheoretische und eine produktivitätsorientierte: Mit wachsenden Einkommen stoße erstens die Nachfrage nach industriellen Produkten an Sättigungsgrenzen, während die nach Dienstleistungen unbegrenzt zunehme. Wegen des geringen Produktivitätsfortschritts der Dienstleistungen müsse daher zweitens der tertiäre Sektor eine überdurchschnittlich steigende Zahl von Arbeitskräften beschäftigen, um diesen wachsenden Bedarf zu befriedigen.

\section{Unstillbarer »Hunger nach Tertiärem«?}

Die Begründung geht also aus von Annahmen über einen gerichteten Wandel der Nachfrage sowie von einer Hierarchie der menschlichen Bedürfnisstruktur: Daraus soll sich bei steigendem Einkommen ein unbegrenzter »Hunger nach Tertiärem« (Fourastié) ergeben. Es werde,

»wie schon der deutsche Statistiker Christian Engel in der zweiten Hälfte des 19. Jahrhunderts feststellte, mit steigendem Volkseinkommen der Anteil der Ausgaben für die häusliche Ver-

1 Auf marxistische Definitionsversuche von Dienstleistungen als »unproduktive Arbeit (vgl. z.B. Mandel 1972), kann in diesem Rahmen nicht eingegangen werden. Aufgrund dieser Definition eine notwendige innere Grenze der Tertiarisierung in kapitalistischen Gesellschaften zu postulieren, scheint aber kaum weniger fragwürdig, als umgekehrt ein immerwährendes Wachstum der Dienstleistungen zu behaupten (vgl. dazu Rohwer 1991). 
pflegung geringer und der Überschuß zunächst in Gebrauchsgüter (wie Kleidung, Wohnung, Auto) und dann in Luxusartikel, Erholung und dergleichen gesteckt« (Bell 1975, 135).

Die Schwächen und Widersprüche dieses nachfragetheoretischen Begründungsversuchs sind mehrfach dargestellt worden (vgl. Voss 1976; Rasmussen 1977; Heinze 1979; Gershuny 1981; Polster/Voy 1989; Polster 1991). Zunächst läßt sich empirisch zeigen, daß die Expansion des tertiären Sektors in der Bundesrepublik Deutschland seit 1945 sich nur zu einem äußerst geringen Teil der Dynamik der privaten, marktvermittelten Nachfrage nach Dienstleistungen verdankt hat. Das überdurchschnittliche Wachstum wurde im wesentlichen vom Staat getragen, und bei den um den Staat bereinigten Dienstleistungen lag der Wachstumsschwerpunkt vor allem bei den produktionsorientierten Diensten (wie Verwaltung, Forschung, Finanzwesen, Transport, Informationsübertragung) (vgl. Heinze 1979, 12 ff.). Ein wesentliches Motiv für die wachsende Nachfrage von Unternehmen nach Dienstleistungen waren Rationalisierungen der industriellen Produktion. Demgegenüber ist der Anteil der Dienstleistungen am privaten Verbrauch zwischen 1960 und 1986 nur mäßig gestiegen, der der personenbezogenen Dienste, auf denen die Hoffnungen der post-industriellen Theoretiker ruhen, sogar leicht gefallen (Polster 1991, 220 f.). ${ }^{2}$ Dies läßt den Schluß zu,

»aß in der Drei-Sektoren-Hypothese die Bedeutung der Nachfrage der privaten Haushalte als strukturbestimmender Faktor insgesamt wie auch als Determinante für das Wachstum der Dienstleistungszweige überschätzt wird « (Heinze 1979, 14).

Zudem übersieht das Nachfrage-Argument, daß ein Anwachsen des tertiären Wirtschafts-Sektors keineswegs mit einer absolut steigenden Versorgung der Gesellschaft mit Dienstleistungen einhergehen muß. Dieses Wachstum kann vielmehr einerseits aus der rationalisierungsbedingten Auslagerung tertiärer Funktionen aus der unmittelbaren Produktion, andererseits aus der »Substitution bisher unentgeltlich erbrachter Hilfe durch kommerzielle und öffentliche« resultieren (Gross 1983, 39). Letzteres trifft vor allem für die Expansion vieler staatlich finanzierter Dienste, vor allem im Sozial-, Gesundheits- und Pflegebereich zu. Das Wachstum solcher Dienstleistungszweige, etwa des Gesundheitswesens, verdankt sich einem Komplex sehr heterogener Ursachen, von der Steigerung der Lebenserwartung über das Auftreten neuer Krankheitsbilder bis hin zur Verbesserung und Intensivierung der medizinischen Technik (vgl. Polster 1991, 242). Es läßt sich kaum eindimensional auf eine gestiegene gesellschaftliche Nachfrage nach $»$ Gesundheit« zurückführen und korrespondiert durchaus nicht

2 Für Großbritannien zeigt Gershuny (1981: 87 ff.), daß die Ausgaben der privaten Haushalte für Dienstleistungen zwischen 1954 und 1974 trotz deutlich gestiegener Einkommen konstant geblieben sind, da zahlreiche Dienstleistungen durch industrielle Produkte ersetzt worden sind. zwangsläufig mit einem insgesamt verbesserten Gesundheitszustand der Bevölkerungen der industriell entwickelten Länder. Vielmehr übernimm ein großer Teil des tertiären Bereichs zunehmend die Funktion, die gesundheitlichen, sozialen, ökologischen Folgeprobleme der industriell-kapitalistischen Modernisierung zu bearbeiten. ${ }^{3}$ In jedem Fall werden die Hoffnungen auf das postindustriell verheißene »schöne Leben« (Bell) durch Gesundheit und Bildung begleitet von der nicht unrealistischen

»Befürchtung, die überproportionale Zunahme des Dienstleistungshereichs, und insbesondere der Dienstleistungen von Mensch zu Mensch (im sozialstaatlichen Bereich, P.W.), schwäche möglicherweise die vorinstitutionellen Ausgleichssysteme und sei gleichbedeutend Kommerzialisierung und Vermachtung der Primärgruppenbezüge« (Gross 1983, 151).

Gravierender noch ist, daß die lineare nachfragetheoretische Begründung der Tertiarisierung eine gerade seit 1945 in vielen Bereichen dominante, gegenläufige Tendenz der Ersetzung von Dienstleistungen durch Warenkonsum übersieht. Das signifikanteste - gerade unter ökologischen Aspekten äußerst folgenreiche - Beispiel dafür ist der Triumphzug des Automobils gegenüber öffentlichen oder kommerziellen Verkehrs-Dienstleistungen. ${ }^{4}$ Weitere bekannte Beispiele sind die Verbreitung der Haushaltstechnik, des Fernsehens und der Unterhaltungselektronik, die zur Schrumpfung entsprechender Dienstleistungsbereiche, etwa der Kinos, entscheidend beigetragen hat.

Im privaten Verbrauch hat sich seit 1945 der Kauf industriell erzeugte Waren als das dominierende Element erwiesen, gegenüber dem der Anteil der Ausgaben für Dienstleistungen sich nur bescheiden erhöhte. Die nachfragetheoretische Begründung für das Wachstum des Dienstleistungssektors überschätzt nicht nur generell die Bedeutung des privaten Konsums sie begeht vor allenm den Fehler, bei steigenden Durchschnitts-Einkommen von einer vom Preis bzw. den Produktionskosten unbeeinflußten Nachfrage nach »Tertiärem« auszugehen. Auch die symbolische Bedeutung des Besitzes von Waren, der den »aristokratischen « Genuß von Dienstleistungen als Statusmerkmal weitgehend abgelöst hat, wird von der Postindustrialismus-Theorie verkannt. So kommen ihr wechselseitige Substitutionsprozesse zwischen industriellem und tertiärem Sektor, zwischen

3 Eine Sonderstellung nimmt in gewisser Hinsicht die Expansion des Bildungssystems ein, deren soziale Dynamik sich nur zum Teil aus funktionalen Imperativen der industrielle Produktion oder aus Professionsinteressen erklären läßt, sondern auch Ausdruck gesellschaftlicher Nachfrage nach Bildung ist. Gleichwohl war der starke Ausbau des Bildungswesens in den 60er und 70er Jahren in der Bundessepublik auch Folge eines ökonomisc . Stagnationsten-

4 Gerade dieses Beispiel verdeutlicht auch, wie schwierig es ist, diesen Trend zu revidiere und den ökologisch und sozial verträglicheren Verkehrs-Dienstleistungen wieder größeres Gewicht gegenüber dem »motorisierten Individualverkehr« zu verschaffen. 
vorinstitutionellen, staatlichen und kommerziellen Dienstleistungen sowie die (Rück-)Verlagerung von Diensten in den Bereich unbezahlter, häufig von Frauen zu erbringender Arbeit nicht mehr in den Blick. Gerade der Verweis auf die Beobachtungen von Engel schließt schematisch und fälschlich vom Luxus-Konsum privilegierter Schichten mit hohen Einkommen auf den Massenkonsum bei allgemein langsam steigenden Einkommen (vgl. Lutz 1989, 216f.; Scharpf 1986, 4f.). Entscheidend für den Konsum von Diensten ist nicht die absolute Höhe der Einkommen, sondern das Ausmaß3 der gesellschaftlichen Einkommensdifferenzierung. Fourastiés und Bells Visionen bleiben einem aristokratischen Konsumideal des 19. und frühen 20. Jahrhunderts verhaftet; die expansiven Potentiale des massenhaften Warenkonsums und der diesem entsprechenden Lebensweisen sind von ihnen kaum erkannt worden (Polster 1991).

Produktivitätsrückstand der Dienstleistungen?

Die zweite Begründung für ein kontinuierliches Wachstum des tertiären Sektors geht ebenfalls auf Fourastié zurück und behauptet, daß Dienstleistungen zu einem weit geringeren Maß rationalisierbar sind als industrielle (und auch landwirtschaftliche) Tätigkeiten. Bei Fourastié fungiert die Unzugänglichkeit für den technischen Fortschritt wie gesehen als Abgrenzungskriterium des tertiären Sektors - womit die These vom Produktivitätsrückstand im Grunde tautologisch wird. Unterstellt man jedoch diesen Rückstand als Tatsache, dann müßten bei steigender industrieller Produktivität immer mehr Arbeitskräfte zur Befriedigung einer wachsenden Nachfrage nach Dienstleistungen in den tertiären Sektor überwechseln.

Begründet wird die These vom Rationalisierungsrückstand (wenn nicht schon definitionsgemäß vorausgesetzt) zumeist mit der Struktur der (vor allem personenbezogenen) Dienstleistungen, mit dem sogenannten »Unoactu-Prinzip« eines zeitlich-räumlichen Zusammenhangs der Produktion und Konsumtion von Diensten:

》Jedenfalls bei den personenbezogenen Dienstleistungen setzt die Erbringung wenigstens die Anwesenheit und oft sogar die aktive Mitwirkung des Klienten voraus. (...) Angesichts der zeitlich schwankenden Inanspruchnahme sind Überkapazitäten auf der Anbieter-Seite erforderlich, die einer Durchrationalisierung der Dienstleistungsproduktion entgegenstehen. (Scharpf 1986, 15; vg1. auch Offe 1984, 237ff.)

Vor allem die neuen elektronischen Medien bieten aber, durch zuvor ungeahnte Möglichkeiten der Speicherung von Diensten und Informationen, zahlreiche Ansatzpunkte, das »Uno-actu-Prinzip« aufzuheben, Produzentenzeit und Konsumentenzeit - zeitlich und räumlich - zu entkoppeln und damit neue Rationalisierungsperspektiven zu eröffnen. Unter dem dadurch entstehenden Rationalisierungsdruck verschärfen sich Tendenzen, gerade die weiterhin an das »Uno-actu-Prinzip« gebundenen Dienste wegen zu hoher Kosten aus dem Markt zu verdrängen und zu privatisieren (vgl. Scharpf 1986). Zugleich haben die neuen mikroelektronischen Technologien einen erheblichen Teil der Dienstleistungstätigkeiten, insbesondere in Verwaltungen, Banken, Versicherungen etc., einem »bis dato unvorstellbaren Rationalisierungsdruck « ausgesetzt und damit die Annahme eines prinzipiellen Rationalisierungsrückstandes als »Illusion« herausgestellt (Baethge / Oberbeck 1986, 18; vgl. Höflich-Häberlein/Häbler 1989, 73).

Offenbar gilt die These vom schwachen technischen Fortschritt nur für einen kleinen und in seiner Bedeutung abnehmenden Teil des tertiären Sektors, vor allem für viele personenbezogene Dienste. Bezeichnenderweise ist Fourastiés (1954, 64f.) Paradebeispiel für den Produktivitätsrückstand die relativ rationalisierungsgeschützte Tätigkeit des Friseurs. Als generelles Charakteristikum von Dienstleistungsarbeit ist das Kriterium der Rationalisierungsgrenzen dagegen kaum aufrechtzuerhalten (vgl. Hönekopp / Ullmann 1980).

In fast allen Prognosen und Diagnosen der Postindustrialismus-Theoretiker finden sich darüber hinaus zwei »blinde Flecken«: die internationale und die geschlechtliche Arbeitsteilung. Generell vernachlässigen die Postindustrialisten, inwieweit die Verschiebung zu den Dienstleistungen in den entwickelten Gesellschaften durch die Strukturen der internationalen Arbeitsteilung mitbedingt ist und wie sie auf diese zurückwirkt (vgl. dazu Heinze 1979; Hönekopp/Ullmann 1980). Das Wachstum des tertiären Sektors in den industriellen Metropolen ist zum Teil Ursache, aber auch Folge der Verlagerung arbeitsintensiver Industrieproduktionen in »Billig-Lohn-« und »Schwellenländer«. Schon aus diesem Grund bleibt zweifelhaft, ob mit den sektoralen Beschäftigungsverlagerungen der Verbrauch von Industrieprodukten in den »nachindustriellen Gesellschaften« überhaupt porportional abnimmt oder nicht vielmehr durch wachsende Importe gedeckt wird.

Auch die Bedeutung der geschlechtlichen Arbeitsteilung als eigener Dimension sozialer Hierarchie, die sektorale Differenzierungen übergreift, wird von den Dienstleistungstheoretikern leicht übersehen. Diese steht in doppelter Hinsicht in Wechselwirkungen mit den sektoralen Verschiebungen: Auf der einen Seite schlagen sich nicht wenige Verlagerungen von Dienstleistungen zum Warenkonsum (etwa von der Wäscherei zur Waschmaschine) in unbezahlter häuslicher Mehrarbeit nieder, die in der Regel von Frauen zu leisten ist. Auf der anderen Seite reproduziert und erneuert sich in der Ausweitung der Dienstleistungstätigkeiten auch eine geschlechtliche Segmentierung der Erwerbsarbeit: Überdurchschnittlich häufig sind schlecht bezahlte, ungeschützte oder im sozialen Aufsteig blockierte (Teilzeits-)Arbeitsplätze im Dienstleistungsbereich von Frauen besetzt (vgl. Baethge/Oberbeck 1986; Mutz 1991). 


\section{Stagnationstendenzen der Tertiarisierung}

Beide Begründungen eines gleichsam evolutionären Übergangs zur nachindustriellen Gesellschaft: die unaufhaltsame Verschiebung der Nachfrage zu den Dienstleistungen sowie der Rationalisierungsrückstand tertiärer Tätigkeiten, lassen sich weder theoretisch unumschränkt aufrechterhalten noch empirisch nachweisen. Somit bezieht die Theorie ihre Plausibilität vor allem aus der Evidenz des schnellen Wachstums der Dienstleistungen in den entwickelten industriellen Gesellschaften nach 1945.

Seit Mitte der 70er Jahre jedoch haben ein starker Rationalisierungsschub, Stagnationstendenzen in der Nachfrage nach Dienstleistungen sowie vor allem Reduzierungen der staatlichen Sozialausgaben zu einem $\gg$ Entwicklungsbruch « geführt, der die expansive Dynamik des tertiären Sektors deutlich abgeschwächt hat. Auch wenn die Beschäftigungssituation in Teilen des Dienstleistungsbereichs weniger konjunkturabhängig ist als in der industriellen Produktion und sogar noch Arbeitsplatzgewinne zu verzeichnen sind, gehören die hohen Wachstumsraten dennoch der Vergangenheit an. Das bedeutet, daß der tertiäre Sektor die ihm zugewiesene und bis Mitte der 70er Jahre auch wahrgenommene $»$ Funktion der Kompensation für Beschäftigungseinbrüche im primären und sekundären Sektor in Zukunft nicht mehr erfüllen wird« (Baethge/Oberbeck 1986, 312; vgl. Mutz 1987). Vielmehr scheint in den industriell entwickelten Ländern ein Zustand erreicht, bei dem $»$ die großen Umschichtungen zwischen den Sektoren weitgehend abgeschlossen sind « (Müller 1983, 152).

Grundsätzlich vernachlässigt der Verweis auf den kontinuierlichen Beschäftigungszuwachs im Dienstleistungsbereich zwei Umstände: zum einen die funktionale Verflechtung des tertiären Sektors mit der industriellen Produktion, zum anderen seine strukturelle Abhängigkeit von kapitalistischer Rentabilität sowie von staatlicher Finanzkraft (die wiederum eng an ökonomische Prosperität gebunden ist).

Wie eng diese funktionale und strukturelle Verflechtung mit der industriell-kapitalistischen Produktion war und ist, zeigt eine Differenzierung des Wachstums des tertiären Sektors nach Funktionen und Produktionsleistungen (Müller 1983, 148): Die »Dienste für Produzenten« konnten in der Bundesrepublik ihren Anteil an den Erwerbstätigen von 1950 bis 1980 von 9,7\% auf $18,4 \%$ fast verdoppeln; die $\gg$ Dienste des Personen-, Nachrichten-, Waren- und Geldverkehrs«, die ebenfalls in hohem Maße produktionsbezogen sind, steigerten ihren Anteil von 13,9\% auf 20,1\% ; »öffentliche Verwaltung und Dienste im öffentlichen Interesse « nahmen von $5,8 \%$ auf $11,0 \% \mathrm{zu}$, sowie »wohlfahrtsstaaatlich alimentierte Dienste« (im Bildungsund Gesundheitswesen) von 3,2\% auf 7,9\%. Dagegen ging der Anteil der hauswirtschaftsnahen Dienste, die als einzige fast ausschließlich dem privaten Konsum dienen, von 5,6\% auf 4,4\% zurück. Auch für den Zeitraum von 1977 bis 1988 zeigt eine Untersuchung in zwölf Ballungsräumen der Bundesrepublik einen engen Zusammenhang zwischen der Beschäftigungsentwicklung im industriellen und im Dienstleistungsbereich: Regionen mit den stärksten Arbeitsplatzverlusten in der Industrie weisen auch die niedrigsten Wachstumsraten im Dienstleistungsbereich auf, und umgekehrt verzeichnen die Ballungsräume mit den geringsten Verlusten oder gar $\mathrm{Ge}$ winnen im industriellen Bereich auch die höchsten tertiären Zuwachsraten (Reissert u.a. 1989).

Die expansive Dynamik des tertiären Sektors nach 1945 läßt sich vor diesen Hintergrund kaum als Ausdruck eines evolutionären, unaufhaltsamen Trends in »modernen « Volkswirtschaften deuten; vielmehr stellt sie das Resultat einer historisch bestimmten und begrenzten Entwicklungs- und Prosperitätsphase industriell-kapitalistischer Gesellschaften dar. Die Abschwächung dieser Dynamik wäre dann als Folge der Erschöpfung dieser Prosperitätskonstellation zu interpretieren (vgl. Lutz 1989: 228ff.). Das heißt auch, daß die mit der Dienstleistungsgesellschaft verknüpfte Vorstellung der »Vollbeschäftigung « aufgrund der »Auffangtätigkeit« des tertiären Sektors vermutlich auf längere Zeit verabschiedet werden muß.

Charakteristisch für die bis Mitte der 70er Jahre anhaltende Prosperitätsphase waren nach Lutz (1989) vor allem

- eine enorme Zunahme und Ausweitung der industriellen Produktion, die auch zu einem hohen Bedarf an produktionsbezogenen Dienstleistungen geführt hat;

- eine bis dahin unbekannte Erhöhung des Lebensstandards und Ausweitung des Massenkonsums sowie daraus resultierend dramatische Veränderungen der Lebensweisen in den industriellen Gesellschaften;

- die Durchsetzung einer wohlfahrtsstaatlichen Politik in allen entwickelten kapitalistischen Ländern, die sich in der beschleunigten Ausweitung staatlicher Dienste niedergeschlagen hat;

die industriell-kapitalistische Absorption des »traditionellen« Wirtschaftssektors Handel, Handwerk, Dienstleistungen, Landwirtschaft und die Mobilisierung der darin, häufig in Familienbetrieben, gebundenen billigen Arbeitskräfte;

damit zusammenhängend die Transformation vieler Tätigkeiten des traditionellen Sektors in kommerzielle Dienstleistungen, die auf diese Weise aber marktwirtschaftlichen Rentabilitätskalkülen und starkem Rationalisierungsdruck unterworfen werden (was wiederum zu ihrer Reprivatisierung und/oder Substitutierung durch Industrieprodukte führen kann). 
Dies macht deutlich, daß Fourastiés aus dem Jahre 1949 stammende Definition des tertiären Sektors als eines kaum rationalisierbaren ProduktionsBereichs gebunden bleibt an eine weitgehend traditionelle, vorindustrielle Form der Erbringung von Dienstleistungen. Der tertiäre Sektor umfaßt nach Fourastié $(1954,80)$ »m wesentlichen den Handel, die Verwaltung, das Unterrichtswesen, die freien Berufe und eine große Zahl von Handwerksberufen « - damit zum großen Teil Bereiche, in denen kurz nach dem Ende des 2. Weltkrieges die Formen kapitalistischer Modernisierung noch kaum Fuß gefaßt hatten.

\section{Nachandustrielle Humanisierung der Gesellschaft?}

So fragwürdig wie die theoretische Konstruktion insgesamt bleibt die dem Übergang zur postindustriellen Gesellschaft zugeschriebene Tendenz zur Humanisierung der Gesellschaft. Wie erwähnt, beinhaltet die Expansion eines tertiären Wirtschafts- oder Staatssektors gerade im Bereich der personenbezogenen Dienste oft nur die Ersetzung vorinstitutioneller Strukturen und sozialer Netze durch kommerzielle oder administrative Tätigkeiten. Somit breitet sich im Zuge der Tertiarisierung unter Umständen weniger eine humane Perspektive auf neue gesellschaftliche Tätigkeitsfelder aus, als umgekehrt bürokratische und kommerzielle Bewertungskriterien in Handlungszusammenhänge eindringen, die bis dahin davon nicht berührt waren (vgl. Gross 1983, 41).

Grundsätzlich stützt sich die Behauptung eines humanisierenden Charakters der Dienstleistungen sehr selektiv nur auf einen kleinen Teil von Diensten, nämlich die unmittelbar personenbezogenen. Deutlich wird dies, wenn Bell Dienstleistungen als ein »Spiel zwischen Personen « beschreibt. Personenbezogene Dienstleistungstätigkeiten stellen indessen, abgesehen von der Ambivalenz ihrer Vermarktung oder Verstaatlichung, keineswegs den Hauptanteil an der Expansion des tertiären Sektors. Dagegen lassen sich die expansiven produktionsbezogenen Dienste, ebenso wie die meisten Verkehrs- oder Handelsberufe nur mit Mühe als ein »Spiel zwischen Personen« interpretieren. Vielmehr zeigt sich, verstärkt durch betriebliche Rationalisierungsschübe, »daß die berufliche Zugehörigkeit zum Dienstleistungssektor keineswegs vor Sinnentleerung, Monotonie und anderen Ausdrucksformen von Entfremdung schützt《 (Baethge/Oberbeck 1986, 17).

Bleibt schon 'mikrosoziologisch', innerhalb der Dienstleistungstätigkeiten, das humanisierende Potential zumindest fragwürdig, so noch mehr in der Vision einer Gesellschaft, in deren Mittelpunkt die kommunikative Begegnung zwischen Menschen, das »eigentlich Menschliche« (Fourastié), stehen soll:
\Das hervorstechendste Merkmal der heutigen Arbeitspraxis ist eine wachsende Distanzierung des Menschen von der Natur, aber auch von Maschinen und Geoenständen, und eine Hinwendung zu seinesgleichen ...«(Bell 1975, 375).

Touraine $(1985,326)$ zufolge verlagert sich im Lauf der Geschichte

»das geselischaftliche Leben aus dem Reich der Reproduiktion, das von Ethnologen erforsch wird, in das Reich der Produktion, das für die meisten modernen Gesellschaften charakteristisch ist, und danach in das Reich der Kommunikation, in dem die Wesenszijge eines gesellschaftichen Systems als Beziehungen und Kommunikationsvoreänge zwische cines gesellsche sozialen Hand ungstragern, und nicht mehr langer als Transor Technologie efraßt werden«. Die Gesellschaft werde unabhängig von ihrem »Verhältnis z einer nicht-geselischaftlichen Umwelt«.

Mit der »Abkehr von Natur und Gegenständen«, glaubt Bell $(1975,37)$, schwinden auch die "Zwänge der Vergangenheit«. Für ihn wie für Fourastié liegen das Ziel und das Wesen des technischen Fortschritts darin, »dem Menschen zur Selbstbestimmung und Selbstverwirklichung zu verhelfen « (Fourastié 1954, 309). Die »moderne Maschine « führe zu einer »Spezialisierung des Menschen auf das eigentlich Menschliche« (ebd., 304). Technik und Technologie stellen in dieser idealistischen Auffassung keine Vermittlung menschlich-gesellschaftlicher Zwecke mit Natur dar, sondern eine reine Entäußerung menschlichen Geistes und Willens. Fortgeschrieben wird damit das industrielle Verständnis von Technik, das auf die Herrschaft des menschlichen Geistes über die Natur zielt und sich bis zu frühen Theoretikern der »Industriegesellschaft« wie Saint-Simon zurückverfolgen läßt.

Der Rest von »Fremdheit«, von »Entfremdung«, der dic Widerständigkeit von Natur in den technischen Konstruktionen verkörpert, soll in der kommunikativen Dienstleistungsgesellschaft zum Verschwinden gebracht werden. Aus dem industriellen 》Spiel gegen eine technisierte Natur « soll ein postindustrielles »Spiel《 ohne die Natur werden: »Wer stößt heute bei der Arbeit schließlich noch auf die Natur als etwas Fremdes oder auch Wohltätiges?«, fragt Bell $(1975,375)$ - globale Gefährdungen der natürlichen Lebensgrundlagen demonstrieren unmißverständlich, wie illusionär eine solche Vorstellung ist.

Die Vision der Dienstleistungsgesellschaft beruht nicht auf einer Neubestimmung und Transformation der krisenhaften gesellschaftlichen Naturverhältnisse in industriellen Gesellschaften; sie verlängert vielmehr die Selbstbezüglichkeit der kapitalistischen Ökonomie und den Produktivismus der industriellen Technologie. Damit stellt die $\gg$ Spezialisierung auf das ei-

5 Kommunikation ist demnach das Grundelement nachindustrieller GeselIschaften. Mit solchen Überlegungen sind die Theorien des Postindustrialismus anschlußfähig an die gegenwärtig (zumindest in der Bundesrepublik) dominierenden soziologischen Grundlagentheorien, worin Geselischaft als sinn- und informationsverarbeitender Kornmunikationszusammenhang thematisiert wird (vgi. Becker/Wehling 1993). 
gentlich Menschliche«, die Abkehr von der Natur und den Dingen, auch keine Vision einer humanen Gesellschaft dar: »Gleichsetzung der Humanisierung der Gesellschaft mit ihrer 'Denaturalisierung' ist kein selbstverständlicher Bestandteil gesellschaftlichen Fortschrittsdenkens mehr«. (Gross 1983, 41) Die Abkehr von den Dingen raubt den Menschen die Erfahrung des Anderen, des Nicht-Menschlichen - und droht genau dadurch inhuman zu werden. Denn »dinghaft Entmenschlichtes (ist) Bedingung von Humanität, während Gleichgültigkeit für die Dinge, die als reine Mittel eingeschätzt und aufs Subjekt reduziert werden, Humanität abtragen« hilft (Adorno 1970, 192).

\section{Offes funktionalistischer Rettungsversuch der postindustriellen Utopie}

Mit einem funktionalistischen Ansatz hat Claus Offe (teilweise zusammen mit Johannes Berger) in den 80er Jahren versucht, das Konzept der Dienstleistungsgesellschaft zu reformulieren und dabei seine beiden deutlichsten Mängel zu korrigieren: einerseits die bloß residuale und negative Definition von Dienstleistungen, andererseits die selektive und begrenzte Bindung ihres normativen Gehalts an die personenbezogenen Dienste. Mit einer einheitlichen, funktionsbezogenen Definition von Dienstleistungsarbeit wird die Rettung des utopischen Gehalts der Dienstleistungsgesellschaft, insbesondere unter den Bedingungen der ökologischen Krise, verbunden. Offe $(1984,295)$ schlägt vor,

»einen positiven soziologischen Begriff von Dienstleistungsarbeit zu konstruieren, der auf dic gesellschaftlichen Funktionen abstellt, welche durch Dienstleistungen erfüllt werden können und müssen«. Danach wären Dienstleistungen alle Tätigkeiten, »die auf die Gewährleistung

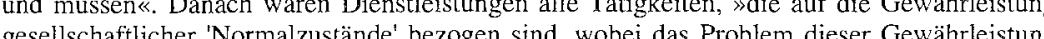
gesellschaflicher 'Non wastan sich darstellt als das des Schutzes und der Bewahrung der ausdifferenzierten Elemente der So-
zialstruktur und der Vermittlung zwischen ihnen« (ebd.).

Abgesehen von dem eigentümlichen Problem, den »Normalzustand « einer Gesellschaft definieren zu müssen, verschiebt dieser Versuch nur die Schwierigkeiten aller früheren Bemühungen, angesichts der Heterogenität von Dienstleistungen ein einheitliches Kriterium festzulegen. Im Anschluß an die geläufige Unterscheidung von Lockwood bezieht Offe »Normalisierungsarbeit « sowohl auf Probleme der Systemintegration als auch auf solche der Sozialintegration (ebd., 298). Damit wird die Unvergleichbarkeit von Tätigkeiten, die auf die Gewährleistung der Funktionskreisläufe des ökonomischen Systems bezogen sind (etwa im Kredit- und Verkehrswesen oder in Industrieverwaltungen) mit Arbeiten, die auf die Vermittlung und Bewahrung sozialer Normen gerichtet sind (etwa im Erziehungs- und Bildungswesen), unter dem Kriterium einer funktionalistischen Meta-Rationa- lität nur verdeckt. Der Begriff »Normalzustand « soll sowohl das normale Funktionieren des Gesellschaftssystems unter systemintegrativen als auch die normalen Wertvorstellungen der Gesellschaftsmitglieder unter sozialintegrativen Gesichtspunkten umfassen - Normalität bezeichnet bloße Faktizität und zugleich soziale Normativität.

Wenn Offe schließlich die auf die Gewährung der (normalen) Bedingungen produktiver Arbeit bezogene Dienstleistungstätigkeit als »Meta-Arbeit « oder »reflexive Arbeit« bezeichnet, enthält diese Definition eine ungeklärte Doppelbedeutung des Begriffs $\gg$ reflexiv $\ll: 6$ Er bezeichnet auf der einen Seite Reflexivität als bloße Selbstbezüglichkeit, als funktionsorientierte Anwendung systemischer Rationalität auf systemische Rationalität; daraus soll auf der anderen Seite eine neue Qualität von Selbst-Reflexion im Sinne einer eigenen reflexiven, nicht mehr bloß instrumentellen Rationalität hervorgehen. Diese wäre gleichsam die »Wiederkehr des Verdrängten «:

$\gg$ Die mit der Verwandlung der Arbeitskraft in eine marktgängige 'Ware' so erfolgreich aus der unmittelbaren Produktion verdrängten Spuren normgeleiteter 'materialer' Rationalität würden sich einer solchen Deutung zufolge ihre Unverzichtbarkeit gleichsam in Gestalt wachsender Dienstleistungsstäbe und -professionen einklagen, deren Aufgabe und 'Arbeit sui generis' darin besteht, die Funktion institutioneller Bestandssicherung durch einen besonderen Typus von Arbeit zu rekonstruieren« (Offe 1984, 26)

Als reflexive Arbeit soll Dienstleistungsarbeit insgesamt eigene qualitative Rationalitätskriterien und Wertmaßstäbe gewinnen, die sie denen der industriellen »Arbeitsgesellschaft « entgegensetzt. Die

»in 'nachindustriellen' Gesellschaften sich herausbildende Gliederung des Gesamtarbeiters in 'Produzenten' und 'Produzenten der Produktion' (...) läßt (...) zwischen den Trägern jener beiden Typen von Arbeit die Rationalitätsmaßstäbe selbst strittig werden, welche den Stoffwechsel mit der Natur lenken (und gegebenenfalls begrenzen) sollen« (ebd., 26)

Aus der funktionalistischen Vereinheitlichung von Dienstleistungen zur »Normalisierungsarbeit« werden unmittelbar Kriterien einer Rationalität sozialer und ökologischer Bewahrung abgeleitet, die zugleich eine neue soziale »Konfliktlinie « konstituieren: In postindustriellen Gesellschaften stünden sich dann die »miteinander unversöhnten Rationalitätskriterien « des »effizienten Herstellers « und der »effektiven Bestandssicherung « ge-genüber - eine plakative Zuschreibung von Bewußtseinslagen in ökologischen Konflikten zu sektoralen Gruppen oder sozialen Klassen (s. dagegen zum ökologischen Bewußtsein von Industriearbeitern Heine/Mautz 1989). Ohnehin sind die Auswirkungen der sektoralen Umschichtungen auf die Sozialstruktur weit geringer als die Theorien der nachindustriellen Gesellschaft, etwa in Bells Vision einer zukünftigen »Wissensgesellschaft«, sug-

6 Dies verbindet Offes Ansatz mit dem Konzept »reflexiver Modernisierung «, wie es in der Bundesrepublik vor allem Ulrich Beck $(1986 ; 1993)$ formuliert hat (vgl. zur Kritik Wehling 1992). 
gerieren. Stattdessen wird die soziale Schichtung auch in $\gg$ Dienstleistungsgesellschaften« eher von Kontinuität und Stabilität geprägt als von revolutionären Umbrüchen (Haller 1982). Leitet man die soziale Lage und den spezifischen Zusammenhang von »objektiven« Lebensverhältnissen und handlungsleitenden Prozessen der Bewußtseinsbildung nicht schematisch aus der sektoralen Zugehörigkeit ab, sondern berücksichtigt Einkommensverteilung, Bildungsniveau, Stellung in der gesellschaftlichen und beruflichen Hierarchie, so zeigt sich, daß klassenspezifische soziale Lagen die Differenzierung nach ökonomischen Sektoren in weiten Bereichen übergreifen und überlagern.

Auch wenn der Dienstleistungssektor zweifellos einen höheren Anteil an gut bezahlten, hochqualifizierten Arbeitskräften aufweist als der industrielle Produktionsbereich, stellt sich die dichotomische Einteilung des »Gesamtarbeiters « nach der Sektorenzugehörigkeit sowohl nach der Seite der Handlungsorientierungen als auch nach der Seite der Klassenbildung als sozialwissenschaftliche Fiktion heraus: Nicht nur sind soziale Lagen und damit auch grundlegende Interessen und Prozesse der Erfahrungs- und Bewußtseinsbildung für Angehörige des sekundären und des tertiären Sektors in vielen Bereichen ähnlich. Darïber hinaus bestehen innerhalb des Dienstleistungssektors erhebliche Differenzierungen, die es unmöglich machen, ihn als sozial oder gar politisch homogenen Faktor zu behandeln. Tatsächlich gehört »ein nicht unbeträchtlicher Teil der Dienstleistungsberufe zu den gesellschaftlich am stärksten benachteiligten Gruppierungen überhaupt« (Haller 1982, 629) - wie etwa die Reinigungs- und zahlreiche Handels- und Verkehrsberufe.

In Offes Konzeption der nachindustriellen Gesellschaft werden Formen herstellender bzw. bewahrender Rationalität dagegen den ökonomischen Sektoren und den in ihnen Tätigen linear zugeordnet: Die funktionale Rationalität der Sicherung der Produktionsvoraussetzungen soll unmittelbar handlungsleitendes Bewußtsein werden; der »objektive « Gehalt der Dienstleistungsarbeit soll die Denk- und Handlungsformen sozialer Gruppen determinieren (damit wiederholt Offe den Fehlschluß einer mechanistischen Klassentheorie und Industriesoziologie). Er projiziert eine (in sich bereits problematische) analytische Unterscheidung von Rationalitätsformen auf getrennte gesellschaftliche Funktionszusammenhänge und jeweilige »Handlungsfiguren«. Diese Projektion ersetzt die Untersuchung und Thematisierung der kontingenten, an den Erfahrungszusammenhang sozialer Akteure gebundenen Formen von Bewußtseinsbildung, auch der durchaus widersprüchlichen Prozesse der Herausbildung eines ökologischen Bewußtseins.
Einerseits werden damit soziale und ökologische Krisen zu Folgen der industriellen Produktion im unmittelbaren Sinn instrumenteller Naturbearbeitung verengt. Auf der anderen Seite aber sollen die Rationalitäts-Potentiale, die jene krisenträchtige instrumentelle Rationalität korrigieren und begrenzen können, in der reflexiven Struktur weiterer Modernisierung, im Übergang zur nachindustriellen Gesellschaft, bereits angelegt sein - als »List der Geschichte« und »Wiederkehr des Verdrängten«. Die behauptete Reflexivität der gesellschaftlichen Entwicklung entspringt indessen nicht den Bewußtseinsleistungen der handelnden Individuen, sondern erscheint als objektives Strukturelement »reflexiver Modernisierung«.

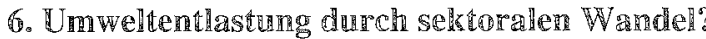

Mit der ökologischen Krise der »modernen Industriegesellschaft« gewannen die Theorien der postindustriellen Gesellschaft in den 80er Jahren neue Anziehungskraft (vgl. neben Offe Touraine u.a. 1982; Jänicke 1985; SRU 1987; Meyer/Ewerhart 1992). Bells Charakterisierung der nachindustriellen Gesellschaft sei, so behauptete ein Theoretiker der »ökologischen Modernisierung «, »zehn Jahre später, im Zeichen der Diskussion um Innovation und Strukturwandel (...) wieder sehr aktuell geworden « (Jänicke 1985, 240). Der sektorale Wandel zur Dienstleistungsgesellschaft und die mit ihm scheinbar verbundenen »ökologischen Gratiseffekte werden in einer solchen Perspektive zu einer Art Königsweg zu einer umweltschonenderen Produktionsweise in den westlichen Gesellschaften.

Die postindustriell-ökologischen Hoffnungen stützen sich vor allem auf einen prognostizierten Wandel in der $\gg$ Rohstoffbasis $«$ der Produktion: Neben dem Wachstum der vermeintlich »sauberen« Dienstleistungen und einer Tendenz zum sparsameren Umgang mit Ressourcen soll es vor allem die intensive Nutzung des »Rohstoffs Information« sein, worauf die ökologische Verträglichkeit der nachindustriellen Ökonomie beruhen werde. Bereits Bell $(1975,12)$ hatte der vor-industriellen Gesellschaft unmittelbare Naturkräfte, der Industriegesellschaft »erzeugte Energie« und der postindustriellen Gesellschaft »Information« als energetische Basis zugeordnet. Daran wurden »zehn Jahre später « kühne Behauptungen geknüpft:

»Das Wachstum einer Informationsökonomie ist (...) ökologisch unbedenklich, ja es könnte den gewünschten Niedergang der 'Schornsteinindustrien' kompensieren « (Jänicke 1985, 257).

Inzwischen werden solche Hoffnungen selbst von ihren Verfechtern wesentlich zurückhaltender bewertet: Die vom sektoralen Wandel erhofften »Gratiseffekte « sind allenfalls in bescheidenem Umfang eingetreten und zudem durch gegenläufige Tendenzen in der Regel wieder neutralisiert 
worden. ${ }^{7}$ Statt »ökologisch cleaner « Dienstleistungsnationen habe sich vielmehr ein »modernisiertes Belastungsmuster « ergeben (Jänicke 1993, 24; vgl. auch Jänicke u.a. 1992). Dieses ernüchternde Resultat verweist über das Scheitern das »sauberen « Postindustrialismus hinaus grundsätzlicher auf die engen Grenzen der Erklärungskraft volkswirtschaftlicher Sektorentheorien gerade in ökologischer Hinsicht. Vor diesem Hintergrund sollen abschließend drei wesentliche Einwände gegen die Hoffnung auf Umweltentlastung durch sektoralen Wandel zusammengefaßt werden: ${ }^{8}$

a) Die Analyse der theoretischen Begründungsversuche für das Wachstum des tertiären Sektors hat ergeben, daß von einer autonomen Ausweitung des Dienstleistungsbereichs nicht die Rede sein kann. Vielmehr bleibt dessen Expansion funktional und strukturell stark abhängig von Wachstum und Produktivitätssteigerung eines hochtechnologischen kapitalistischindustriellen Sektors. Dies räumt die Postindustrialismus-Theorie in ihren Grundannahmen selbst ein, wenn sie die Dynamik des sektoralen Wandels aus steigenden Einkommen und rascher Produktivitätssteigerung im sekundären Sektor erklärt. Tertiarisierung basiert also auf Produktivitätsfortschritt und wachsendem, zumindest aber gleichbleibendem industriellem Produktionsausstoß. In der klassischen Theorie bedeutet Tertiarisierung ohnehin nur die Umschichtung der Beschäftigten zwischen den Sektoren. Daraus folgt aber aufgrund ihrer eigenen Prämissen noch nicht einmal eine relative Verschiebung der Produktionsstruktur hin zu den Dienstleistungen, geschweige denn ein absoluter Rückgang der industriellen Produktion, der unter ökologischen Aspekten in erster Linie relevant wäre (vgl. Hönekopp/Ullmann 1980).

b) Dem ökologischen Postindustrialismus liegt eine latente Gleichsetzung von Industrieproduktion mit »materiell« und »verschmutzend« sowie von Dienstleistungen mit »immateriell« und daher »sauber « zugrunde. Allein

$7 \gg$ Empirische Untersuchungen, die sich mit den Auswirkungen des sektoralen Strukturwandels (...) unter dem Aspekt der Schadstoffbelastung beschäftigen, können die pauwandels (...) unter den Aspekt der Schadston Auswirk beschaftigen, konmen die pauschale These von den Gratiseffekten nicht bestatigen; im Gegenteil, in bestimmten Schadstoffbereichen führte der Strukturwandel zu zusätzlichen Belastungen.« (Ossing u.a. 1991: 342). Hervorgehoben wird dabei auch, das das »sektorale Argumentationsraster zu grob« sei, um umweltrelevante Änderungen der Produktionsformen zu erfassen (RW1 1987)

8 Ein weiterer wichtiger Einwand bezieht sich auf die Ausblendung des internationalen Kontextes der Tertiarisierung auch in ökologisch orientierten Postindustrialismus-Theorien. Tatsächlich kann die Auslagenung umweltbelastender Produktionen in 》BilliglohnLänder globel zu einer Verchärfung ökologischer Problemlagen führen: Einerseits werLän dadurch immer weiere Regionen von indstiellen Schld den dadurch inmer witere Regin wön insting dererseits kann in abhängigen Ländern häufig unter Umgehung der in den westlichen Staaten gültigen Umwelt- und Sicherheitsstandards produziert werden der weiterhin (wenn nicht in den Beschäftigungswirkungen, so doch in den Verkehrsleistungen) äußerst expansive Güterverkehrsbereich demonstriert, daß diese Dichotomie unhaltbar ist. Auch etliche andere DienstleistungsTätigkeiten sind hochgradig »emissionsintensiv«, wie der Sachverständigenrat für Umweltfragen immerhin einräumt. Zudem sind zahlreiche Dienste an hohe industrielle Vorleistungen und materielle Infrastrukturen (etwa der Energieversorgung) gebunden. Auch dies räumt der Sachverständigenrat ein, ohne sich dadurch in seinem Urteil eines »per saldo« umweltentlastenden sektoralen Wandels beirren zu lassen (SRU 1987, TZ 283).

c) Als fast völlig verfehlt haben sich die Prognosen des Postindustrialismus über die Veränderungen der Verbrauchsstrukturen und der Lebensweisen in den entwickelten Industriegesellschaften herausgestellt. Diese gehen weit eher in Richtung einer »Selbstbedienungswirtschaft« (Gershuny 1981) oder des massenhaften »demokratischen Warenkonsums« (Polster 1991) als hin zu einer Intensivierung des Dienstleistungskonsums oder gar der personenbezogenen Dienste, worin das Humanisierungspotential der nachindustriellen Gesellschaft vermutet wurde. Der verallgemeinerte Konsum langlebiger, industriell hergestellter Gebrauchsgüter aber führt sowohl direkt als auch vermittelt über die Ankurbelung der Industrieproduktion zu hohem Ressourcen- und Energieverbrauch und umweltbelastenden Schadstoffemissionen.

Die Entwicklungsdynamik der hoch industrialisierten Gesellschaften seit 1945 ist sowohl im Bereich der Produktion als auch in dem des Konsums, sowohl unter ökonomischen als auch unter sozio-kulturellen und ökologischen Aspekten wesentlich vielschichtiger und widersprüchlicher verlaufen, als es das recht einfache »historische Modell« des sektoralen Wandels zur Dienstleistungsgesellschaft behauptet hatte. Das post-industrielle Deutungsmuster erzeugt ökologisches »falsches Bewußtsein«, wenn es suggeriert, Umweltbelastungen seien in erster Linie eine Folge ohnehin überlebter »Schornsteinindustrien«. Strategien einer technikfixierten »ökologischen Modernisierung « und eines forcierten wirtschaftlichen Strukturwandels zur tertiären »Informationsgesellschaft « erscheinen dann als Wege aus der ökologischen Krise. Doch die scheinbare »Versöhnung « zwischen Wachstumsökonomie, technologischem Fortschritt und Schonung der Natur im ökologischen Postindustrialismus kann allenfalls zu selektiven technischen Lösungen von Umweltgefährdungen führen, denen auf der anderen Seite Problemverlagerungen und -verschärfungen sowie völlig neuartige Gefahrenpotentiale (etwa in der Gentechnik) gegenüberstehen.

Demgegenüber sollte eine ökologisch orientierte Politik die gesellschaftliche Arbeit insgesamt als dominierenden Bereich der Regulierung gesellschaftlicher Naturverhältnisse begreifen und zu gestalten versuchen. Statt 
auf das langsame Verschwinden industrieller Naturbearbeitung in der Dienstleistungsgesellschaft zu setzen, muß sie Perspektiven einer übergreifenden ökologischen Umstrukturierung aller ökonomischen »Sektoren Landwirtschaft, Industrie und Dienstleistungen, entwickeln. In einem solchen Rahmen kann eine gezielte umweltpolitische Strategie der Transformation rein produktbezogener Industrien in integrierte Dienstleistungsfelder - etwa durch die Zurückdrängung des Autos zugunsten öffentlicher Mobilitäts-Dienste - durchaus ihren Stellenwert haben. Dies wäre aber Ausdruck und Ergebnis konfliktreicher gesellschaftlicher Auseinandersetzungen, nicht eines vermeintlich evolutionären Entwicklungstrends.

Die Verringerung des Ressourcen- und Energieverbrauchs von Produktionsvorgängen ist zweifellos ein entscheidendes Element eines solchen »ökologischen Umbaus«. Gleichwohl helfen Visionen einer technischen »Effizienzrevolution« allein wenig weiter; bleiben sie isoliert, laufen sie unter Umständen sogar Gefahr, ein bloß technisches Fortschrittsmuster zu verlängern. Beginnen muß ein demokratischer Prozeß sowohl des Umbaus der materiellen Infrastruktur der Produktion und ihrer sozialen Organisationsformen als auch der Veränderung der seit den 50er Jahren dominant gewordenen Konsummuster und Lebensweisen. Dies schließt ein, Konzepte einer anderen Verteilung von Arbeit zu entwerfen und die Rationalität einer technischen Entwicklung in Frage zu stellen, die eindimensional auf die Marginalisierung menschlicher Arbeitskraft hinausläuft. Denn es spricht wenig dafür, daß der tertiäre Sektor künftig alle die Beschäftigten auffangen kann, die aufgrund industrieller Produktivitätssteigerung »überflüssig " zu werden drohen - es sei denn um den Preis extremer Einkommensdifferenzierungen.

\section{Literatur}

Adorno, Th. W. 1970: Negative Dialektik, Frankfurt-M.

Baethge, M./Oberbeck, H. 1986: Zukunft der Angestellten. Neue Technologien und beruffiche Perspektiven in Büro und Verwaltung, Frankfurt-M./New York

Beck, U. 1986: Risikogesellschaft, Frankfurt-M.

Beck, U. 1993: Die Erfindung des Politischen, Frankfurt-M.

Becker, E./Wehling, P.1993: Risiko Wissenschaft. Ökologische Perspektiven in Wissenschaft und Hochschule, Frankfurt-M./New York

Bell, D. 1975: Die nachindustrielle Gesellschaft, Frankfurt-M./New York

Fourastié, J. 1954: Die große Hoffnung des XX. Jahrhunderts, Köln

Gershuny, J. 1981: Die Ökonomie der nachindustriellen Gesellschaft, Frankfurt-M.New York

Gross, P. 1983: Die Verheißungen der Dienstleistungsgesellschaft. Soziale Befreiung oder Sozialherrschaft?, Opladen

Haller, M. 1982: Auf dem Weg zur Dienstleistungsgesellschaft?, in: Wirtschaft und Gesellschaft, 8. Jg.

Heine, H./Mautz, R. 1989: Industriearbeiter contra Umweltschutz?, Frankfurt-M./New York
Heinze, J. 1979: Strukturwandel in der Bundesrepublik, in: Ifo-Schnelldienst, 32. Jg.. H. 33

Höflich-Häberlein, L./Häbler. H. 1989: Diffusion neuer Technologien und ihre Auswirkungen im privaten Dienstleistungssektor, in: R. Schettkat/M. Wagner (Hg.): Technologischer Wandel und Beschäftigung, Berlin/New York

Hönekopp, E./Ullmann, H. 1980: Auf dem Weg zur Dienstleistungsökonomie?, in: Mitteilungen aus der Arbeitsmarkt- und Berufsforschung, 13. Jg., H. 2

Jänicke, M. 1985: Superindustrialismus und Postindustrialismus - Langzeitperspektiven von Umweltbelastung und Umweltschutz, in: M. Jänicke u.a. (Hg.): Wissen für die Umwelt, Berlin/New York

Jänicke, M. 1993: Zukunftsfähige Entwicklung in Europa?, in: Wechselwirkung, Nr. 61 (15. Jg.)

Jänicke, M./Mönch, H./Binder, M. u.a. 1992: Umweltentiastung durch industriellen Strukturwandel? Eine explorative Studie über 32 Industrieländer 1970 bis 1990), Berlin

Lutz, B. 1989: Der kurze Trauin immerwährender Prosperität, 2., erw. Auflage, FrankiurtM./New York

Mandel, E. 1972: Der Spätkapitalismus, Frankfurt-M.

Meyer, B./Ewerhart, G. 1992: Die »saubere« Dienstleistungsgesellschaft. Eine konsistente Projektion?, Beiträge des Instituts für empirische Wirtschaftsforschung, Nr. 30, Osnabrück

Müller, W. 1983: Wege und Grenzen der Tertiarisierung. Wandel der Berufsstruktur in der Bundesrepublik Deutschland 1950 - 1980, in: J. Matthes (Hg.): Krise der Arbeitsgesellschaft?, Frankfurt-M./New York

Mutz, G. 1987: Arbeitslosigkeit in der Dienstleistungsgesellschaft, in: Soziale Welt, 38. Jg.

Mutz, G. 1991: Auf Umwegen zur Dienstleistungsgesellschaft? Kritische Anmerkungen zum Gehalt postindustrieller Szenarien, in: W. Littek u.a. (Hg.): Dienstleistungsarbeit, Berlin

Offe, C. 1984: »Arbeitsgesellschaft M./New York

Ossing, F./Polster, W./Thomasberger, C./Voy, K. 199l: Innere Widersprüche und äußere Grenzen der Lebensweise - Aspekte der ökologischen Entwicklung, in: K. Voy u.a. (Hg.): Gesellschaftliche Transformationsprozesse und materielle Lebensweise. Beiträge zur Wirtschafts- und Gesellschaftsgeschichte der Bundesrepublik Deutschland 1949-1989, Bd. 2, schafts- $u$ thd
Marburg

Polster, W. 1991: Wandlungen der Lebensweise im Spiegel der Konsumentwicklung - Vom Dienstleistungskonsum zum demokratischen Warenkonsum, in: K. Voy u.a. (Hg.)

Polster, W./Voy, K. 1989: Die Entfaltung der Industriewirtschaft - Zum Strukturwandel von Wirtschaft und Erwerbsarbeit in der Industriegesellschaft, in: K. Voy u.a. (Hg.): Marktwirt. schaft und politische Regulierung. Beiträge zur Wirtschafts- und Gesellschaftsgeschichte der Bundesrepublik Deutschland 1949-1989, Bd. 1, Marburg

Rasmussen, Th. 1977: Entwicklungslinien des Dienstleistungssektors, Göttingen

Reissert, B./Schmid, G./ Jahn, S. 1989: Mehr Arbeitsplätze durch Dienstleistungen? Ein Vergleich der Beschäftigungsentwickiung in den Ballungsregionen der Bundesrepublik Deutschland, WZB-discussion paper FS I 89-14, Berlin

Rohwer, G. 1991: Möglichkeiten und Grenzen einer kapitalistischen Dienstleistungsgesellschaft, Hamburger Institut für Sozialforschung, Discussion Paper 2-91, Hamburg

RWI 1987: Rheinisch-Westfälisches Institut für Wirtschaftsforschung: Strukturberichterstattung 1987. Schwerpunktthema: Strukturwandel und Umweltschutz, Essen

Scharpf, F.W. 1986: Strukturen der postindustriellen Gesellschaft, in: Soziale Welt, 37. Jg.

SRU 1987: Rat von Sachverständigen für Umweltfragen: Umweltgutachten 1987, Mainz

Touraine, A. 1972: Die postindustrielle Gesellschaft, Frankfurt-M.

Touraine, A. 1985: Klassen, soziale Bewegungen und soziale Schichtung in einer nachindustriellen Gesellschaft, in: H. Strasser/J. Goldthorpe (Hg.): Die Analyse sozialer Ungleichheit, Opladen

Touraine, A. u.a. 1982: Die anti-nukleare Prophetie, Frankfurt-M./New York

Voss, G. 1976: Trend zur Dienstleistungsgesellschaft?, Köln

Wehling, P. 1992: Die Moderne als Sozialmythos. Zur Kritik sozialwissenschaftlicher Modernisierungstheorien, Frankfurt-M./New York 